\title{
A DIFFUSION LIMIT FOR GENERALIZED CORRELATED RANDOM WALKS
}

\author{
URS GRUBER, ${ }^{*}$ Allianz $A G$ \\ MARTIN SCHWEIZER, ${ }^{* *}$ ETH Zürich
}

\begin{abstract}
A generalized correlated random walk is a process of partial sums $X_{k}=\sum_{j=1}^{k} Y_{j}$ such that $(X, Y)$ forms a Markov chain. For a sequence $\left(X^{n}\right)$ of such processes in which each $Y_{j}^{n}$ takes only two values, we prove weak convergence to a diffusion process whose generator is explicitly described in terms of the limiting behaviour of the transition probabilities for the $Y^{n}$. Applications include asymptotics of option replication under transaction costs and approximation of a given diffusion by regular recombining binomial trees.
\end{abstract}

Keywords: Correlated random walk; diffusion limit; weak convergence; mathematical finance; large investor; transaction cost; binomial tree

2000 Mathematics Subject Classification: Primary 60F17; 60J60; 91B28

\section{Introduction}

Let $\left(Y_{j}\right)_{j \in \mathbb{N}_{0}}$ be a sequence of random variables and $X_{k}=\sum_{j=1}^{k} Y_{j}, k \in \mathbb{N}_{0}$, the corresponding process of partial sums. If the $Y_{j}$ are independent, $X$ is a random walk and Donsker's theorem shows that, under suitable assumptions and after rescaling, a sequence of such walks $X^{n}$ converges in distribution to a Brownian motion. If the $Y_{j}$ form a Markov chain, then $X$ is a correlated random walk and there are also some relevant weak convergence results; details are given later. In this paper, we study the more general case in which the pair $(X, Y)$ is a Markov chain, and prove a functional central limit theorem for a sequence of such processes $X^{n}$.

More precisely, we consider the situation in which each $Y_{j}^{n}$ takes only two values. The limit process $X^{\infty}$ is a diffusion and we explicitly describe its generator in terms of the limiting behaviour of the transition probabilities for the $Y^{n}$. The motivation for this problem comes from mathematical finance, where it arises in the context of option pricing for a large investor. We briefly sketch this connection and give two other applications of the main convergence result. One is about asymptotics for option replication under transaction costs, and the other shows how we can approximate a given diffusion by a regular recombining binomial tree.

The paper is structured as follows. Section 2 contains the precise set-up and the main result as well as comments on the literature. In Section 3, we prove the main result and discuss variations and extensions. In Section 4, we present the applications.

\footnotetext{
Received 29 June 2005.

* Postal address: Allianz AG, Allianz Global Risks, Königinstrasse 28, D-80802 München, Germany.

Email address: ursgruber@gmx.net

** Postal address: Departement Mathematik, ETH Zürich, ETH-Zentrum, HG G28.2, CH-8092 Zürich, Switzerland.

Email address: martin.schweizer@math.ethz.ch
} 


\section{Set-up and main result}

Our goal in this paper is to prove a weak convergence result for a class of generalized correlated random walks. This section contains the basic set-up, the assumptions, and the main result.

For each $n \in \mathbb{N}$, we start with a probability space $\left(\Omega^{n}, \mathcal{F}^{n}, \mathrm{P}^{n}\right)$ on which we have binary random variables $\left(Z_{k}^{n}\right)_{k=0,1, \ldots, n}$ taking the values +1 and -1 . We define a stochastic process $X^{n}=\left(X_{k}^{n}\right)_{k=0,1, \ldots, n}$ via its increments,

$$
\Delta X_{k}^{n}:=X_{k}^{n}-X_{k-1}^{n}:=\mu_{n}+\sigma_{n} Z_{k}^{n}, \quad k=1, \ldots, n,
$$

where $\mu_{n}$ and $\sigma_{n}$ are constants to be specified later; hence,

$$
X_{k}^{n}=X_{0}^{n}+\sum_{j=1}^{k}\left(\mu_{n}+\sigma_{n} Z_{j}^{n}\right), \quad k=0,1, \ldots, n,
$$

is a sequence of partial sums. Set $t_{k}^{n}:=k / n$. Piecewise-constant interpolation on the intervals $\left[t_{k-1}^{n}, t_{k}^{n}\right)$ yields a process $X^{(n)}=\left(X_{t}^{(n)}\right)_{0 \leq t \leq 1}$ with right-continuous trajectories with left limits, via

$$
X_{t}^{(n)}:=X_{\lfloor n t\rfloor}^{n}, \quad 0 \leq t \leq 1,
$$

where $\lfloor\cdot\rfloor$ is the integer-part function, implying that

$$
X_{t_{k}^{n}}^{(n)}=X_{k}^{n} \quad \text { for } k=0,1, \ldots, n .
$$

We denote by $\mathbb{F}^{(n)}=\left(\mathcal{F}_{t}^{(n)}\right)_{0 \leq t \leq 1}$ the filtration generated by $X^{(n)}$; hence,

$$
\mathcal{F}_{t}^{(n)}=\sigma\left(X_{0}^{n}, X_{1}^{n}, \ldots, X_{k-1}^{n}\right)=: \mathcal{F}_{k-1}^{n} \quad \text { for } t \in\left[t_{k-1}^{n}, t_{k}^{n}\right) \text { and } k=1, \ldots, n .
$$

The distribution of $X^{(n)}$ under $\mathrm{P}^{n}$ is a probability measure $\rho_{n}$ on the Skorokhod space $D[0,1]$ of right-continuous functions with left limits. Our goal is to prove a weak convergence result for the sequence $\left(\rho_{n}\right)_{n \in \mathbb{N}}$, under suitable assumptions on $\mu_{n}, \sigma_{n}$, and the behaviour of $\left(Z_{k}^{n}\right)_{k=0,1, \ldots, n}$ under $\mathrm{P}^{n}$.

Remark 1. It is purely for ease of notation that we work on the time interval $[0,1]$. Analogous results can be obtained for $[0, T]$ with $T \in(0, \infty)$, or for $[0, \infty)$.

Since we are interested in diffusion limits, we work with the usual Donsker-type scaling. Therefore, we let $\delta_{n}:=1 / \sqrt{n}$ and impose the following condition.

Condition 1. There are constants $\sigma>0, \mu \in \mathbb{R}$, and $\beta \in(0,1)$ such that

$$
\begin{aligned}
& \sigma_{n}=\sigma \delta_{n}+O\left(\delta_{n}^{1+\beta}\right), \\
& \mu_{n}=\mu \delta_{n}^{2}+O\left(\delta_{n}^{2+\beta}\right) .
\end{aligned}
$$

Thus, each increment $\Delta X_{k}^{n}$ has mean and variance of the order $\delta_{n}^{2}=1 / n$, as in Donsker's theorem. However, our main assumption is that, under $\mathrm{P}^{n}$, each pair $\left(X^{n}, Z^{n}\right)$ is a Markov chain whose transition probabilities have a suitable form. More precisely, we assume the following. 
Condition 2. We require that

$$
\mathrm{P}^{n}\left[Z_{k}^{n}=1 \mid \mathcal{F}_{k-1}^{n}\right]=p_{n}\left(k, X_{k-1}^{n}, Z_{k-1}^{n}\right) \text { for } k=1, \ldots, n,
$$

where

$$
p_{n}(k, x, z)=\frac{1}{2}\left(1+z a\left(t_{k}^{n}, x\right)+\delta_{n} b\left(t_{k}^{n}, x\right)\right)+O\left(\delta_{n}^{1+\beta}\right)
$$

for $k \in\{1, \ldots, n\}, x \in \mathbb{R}$ and $z \in\{-1,+1\}$.

The assumptions on the functions $a, b:[0,1] \times \mathbb{R} \rightarrow \mathbb{R}$ will be specified presently.

If Condition 2 holds then, under $\mathrm{P}^{n}$, the process $X^{n}$ is a generalized correlated random walk. Suppose that there is no $O$-term in (6). In the simplest case, where $a \equiv 0$ and $b \equiv 0, X^{n}$ is just a binary random walk and Condition 1 implies, via Donsker's theorem, that $\left(X^{(n)}\right)_{n \in \mathbb{N}}$ converges in distribution to a Brownian motion with drift $\mu$ and volatility $\sigma$. If $a \equiv 0$ then $p_{n}$ does not depend on $z$, and $X^{n}$ alone is a Markov chain under $\mathrm{P}^{n}$. If $b \equiv 0$ and $a$ does not depend on $x$, then $Z^{n}$ is an inhomogeneous Markov chain and $X^{n}$ is a cumulative sum of Markovian increments. This is called (in the time-homogeneous case) a correlated random walk; see Chen and Renshaw (1994). The novel feature in the present paper is that the transition probabilities for $Z^{n}$ (or $X^{n}$ ) are allowed to depend on both the current state $X_{k-1}^{n}$ and the current increment $Z_{k-1}^{n}$ (or, equivalently, $\Delta X_{k-1}^{n}$ ). This makes the analysis more delicate and produces more interesting limiting behaviour.

A detailed overview of much of the existing literature on correlated random walks is given in Section 5.1 of Gruber (2004). Hence, we focus here only on papers concerned with weak convergence. Renshaw and Henderson (1981) showed such results for (classical) symmetric correlated random walks, i.e. for $b \equiv 0$ and $a$ constant. These correlated random walks constitute a special type of directionally reinforced random walk for which weak convergence has been analysed by Mauldin et al. (1996) and Horváth and Shao (1998). Szász and Tóth (1984) studied symmetric, and some more general, correlated random walks in a random environment, and weak convergence to Brownian motion for a family of homogeneous correlated random walks has also been obtained by Opitz (1999). However, all these results are for correlated random walks that are homogeneous in time and space; no dependence on the current state is allowed.

Let us now return to our generalized correlated random walks. On the functions $a$ and $b$, which determine the transition probabilities via (6), we impose the following condition.

Condition 3. The functions $a, b:[0,1] \times \mathbb{R} \rightarrow \mathbb{R}$ satisfy

$$
\begin{gathered}
\|a\|_{\infty}:=\sup \{|a(t, x)|,(t, x) \in[0,1] \times \mathbb{R}\}<1 \text { and }\|b\|_{\infty}<\infty ; \\
a^{\prime}(t, x):=\frac{\partial}{\partial x} a(t, x) \text { exists and is bounded on }[0,1] \times \mathbb{R} ; \\
a^{\prime}(t, x) \text { is globally Hölder }(\beta) \text {-continuous in } x, \text { uniformly in } t, i . e . \\
\left|a^{\prime}(t, x)-a^{\prime}(t, y)\right| \leq K|x-y|^{\beta} \text { for all } x, y \in \mathbb{R} \text { and } t \in[0,1] ; \\
b(t, x) \text { is globally Hölder }(\beta) \text {-continuous in } x, \text { uniformly in } t ; \\
a(t, x), a^{\prime}(t, x), \text { and } b(t, x) \text { are all continuous in } t .
\end{gathered}
$$

Without loss of generality, we may and do take the same $\beta \in(0,1)$ for (4), (5), (6), (9), and (10). Under Condition 3, we define the operator $L$ on $C^{2}$ functions $f(x)$ by

$$
(L f)(t, x):=\frac{1}{2} \sigma^{2} \frac{1+a(t, x)}{1-a(t, x)} f^{\prime \prime}(x)+\left(\mu+\frac{\sigma b(t, x)}{1-a(t, x)}+\frac{\sigma^{2} a^{\prime}(t, x)}{(1-a(t, x))^{2}}\right) f^{\prime}(x) .
$$


Our main result is then stated as follows.

Theorem 1. Assume that Conditions $1-3$ hold and that $\left(X_{0}^{n}\right)_{n \in \mathbb{N}}$ converges in distribution to some $X_{0}$ with distribution $v$ on $\mathbb{R}$. If the martingale problem for $L$ is well posed in $C[0,1]$, then $\left(X^{(n)}\right)_{n \in \mathbb{N}}$ converges in distribution to the solution, $X$, to the stochastic differential equation

$$
\mathrm{d} X_{t}=\left(\mu+\frac{\sigma b\left(t, X_{t}\right)}{1-a\left(t, X_{t}\right)}+\frac{\sigma^{2} a^{\prime}\left(t, X_{t}\right)}{\left(1-a\left(t, X_{t}\right)\right)^{2}}\right) \mathrm{d} t+\sigma \sqrt{\frac{1+a\left(t, X_{t}\right)}{1-a\left(t, X_{t}\right)}} \mathrm{d} W_{t},
$$

with initial value $X_{0}$. Here $W=\left(W_{t}\right)_{0 \leq t \leq 1}$ is a (standard) Brownian motion on [0, 1].

A proof of Theorem 1 is given in Section 3; here, we merely make some comments.

A solution to the martingale problem for $(L, v)$ always exists. This follows from Theorem 6.1.7 of Stroock and Varadhan (1979) because Condition 3 implies that both coefficients in $L$ (those of $f^{\prime \prime}(x)$ and $f^{\prime}(x)$ ) are bounded in $t$ and $x$ and continuous in $x$ for each $t$.

If we suppose only that the martingale problem for $(L, v)$ has a unique solution, the conclusion of Theorem 1 is still true. This is not surprising and can be seen from the original proof of Gruber (2004). Even if the martingale problem has no unique solution, we can show that any subsequence of $\left(X^{(n)}\right)$ contains a further subsequence which converges in distribution to some solution of (12). (Of course, the latter need then not be unique.) For more details, we refer the reader to Section 5.3.7 of Gruber (2004).

If $a \equiv 0$ then the proof of Theorem 1 is a straightforward application of Theorem 2.1 of Nelson (1990). However, that result does not extend to $a \not \equiv 0$ because it requires the convergence of the conditional moments $\delta_{n}^{-2} \mathrm{E}\left[\Delta X_{k}^{n} \mid \mathcal{F}_{k-1}^{n}\right]$.

For some applications, it is desirable that the functions $a$ and $b$ also depend on $n$. This is possible within certain limits; see the remark at the end of Section 3.

\section{Proof of the main result}

In this section, we show how to prove Theorem 1 . We do this in detail for the case in which $a$ and $b$ do not depend on $t$, and then explain how to deal with the time-dependent case. We also comment on the original argument of Gruber (2004) and sketch how to extend Theorem 1 to functions, $a_{n}$ and $b_{n}$, depending on $n$. Although this section is the most important contribution of the paper, readers interested only in applications can omit it and proceed directly to Section 4.

We do not prove Theorem 1 from first principles. Our main tool is the following result, which is - up to notational changes - Theorem 7.4.1 of Ethier and Kurtz (1986).

Proposition 1. Let $c: \mathbb{R} \rightarrow[0, \infty)$ and $\gamma: \mathbb{R} \rightarrow \mathbb{R}$ be continuous, define the operator $G$, on $C^{\infty}$ functions $f$ with compact support, by $G f:=\frac{1}{2} c f^{\prime \prime}+\gamma f^{\prime}$, and suppose that the martingale problem for $G$ is well posed on $C[0,1]$. In the setting of Section 2 , let $\Gamma^{(n)}$ and $C^{(n)}$ be $\mathbb{F}^{(n)}$-adapted processes such that $C^{(n)}$ is increasing and both

$$
N^{(n)}:=X^{(n)}-X_{0}^{(n)}-\Gamma^{(n)}
$$

and $\left(N^{(n)}\right)^{2}-C^{(n)}$ are local $\left(\mathrm{P}^{n}, \mathbb{F}^{(n)}\right)$-martingales for each $n$. Set

$$
\tau_{n}^{r}:=\inf \left\{t \geq 0:\left|X_{t}^{(n)}\right| \geq \operatorname{ror}\left|X_{t-}^{(n)}\right| \geq r\right\} \wedge 1
$$


and suppose that, for each $r>0$,

$$
\begin{aligned}
& \lim _{n \rightarrow \infty} \mathrm{E}^{n}\left[\sup _{0 \leq t \leq \tau_{n}^{r}}\left|X_{t}^{(n)}-X_{t-}^{(n)}\right|^{2}\right]=0, \\
& \lim _{n \rightarrow \infty} \mathrm{E}^{n}\left[\sup _{0 \leq t \leq \tau_{n}^{r}}\left|\Gamma_{t}^{(n)}-\Gamma_{t-}^{(n)}\right|^{2}\right]=0, \\
& \lim _{n \rightarrow \infty} \mathrm{E}^{n}\left[\sup _{0 \leq t \leq \tau_{n}^{r}}\left|C_{t}^{(n)}-C_{t-}^{(n)}\right|\right]=0,
\end{aligned}
$$

and

$$
\begin{aligned}
& \sup _{0 \leq t \leq \tau_{n}^{r}}\left|\Gamma_{t}^{(n)}-\int_{0}^{t} \gamma\left(X_{s}^{(n)}\right) \mathrm{d} s\right| \rightarrow 0 \quad \text { in probability as } n \rightarrow \infty \\
& \sup _{0 \leq t \leq \tau_{n}^{r}}\left|C_{t}^{(n)}-\int_{0}^{t} c\left(X_{s}^{(n)}\right) \mathrm{d} s\right| \rightarrow 0 \quad \text { in probability as } n \rightarrow \infty .
\end{aligned}
$$

If the distributions of $X_{0}^{(n)}$ under $\mathrm{P}^{n}$ converge weakly to a probability measure $v$ on $\mathbb{R}$, then $\left(X^{(n)}\right)_{n \in \mathbb{N}}$ converges in distribution to the solution to the martingale problem for $(G, v)$.

The difficulty in applying Proposition 1 is finding the decomposition of a given $X^{(n)}$ into $N^{(n)}$ and $\Gamma^{(n)}$; this is linked in turn to the knowledge of the functions $c$ and $\gamma$ in the generator $G$. One of the main difficulties encountered in Gruber (2004) was to find these functions in the first place, and so a completely different (and much longer) proof was given there. We comment on this below in some more detail.

Because each $X^{(n)}$ and $\mathbb{F}^{(n)}$ is piecewise constant, it is enough to do everything in discrete time. More precisely, we can start with $X^{n}$ and look for processes $Y^{n}=\left(Y_{k}^{n}\right)_{k=0,1, \ldots, n}$, with $Y \in\{N, \Gamma, C\}$, such that $C^{n}$ is increasing and both $N^{n}:=X^{n}-X_{0}^{n}-\Gamma^{n}$ and $\left(N^{n}\right)^{2}-C^{n}$ are $\mathrm{P}^{n}$-martingales for the filtration $\mathbb{F}^{n}:=\left(\mathcal{F}_{k}^{n}\right)_{k=0,1, \ldots, n}$. The corresponding processes $Y^{(n)}$, obtained by piecewise-constant interpolation as in (3), can then be used in Proposition 1. Moreover, the obvious choice for $C^{n}$ is clearly the increasing $\mathbb{F}^{n}$-predictable process from the Doob decomposition of $\left(N^{n}\right)^{2}$; thus, we let

$$
\begin{aligned}
\Delta C_{k}^{n} & =C_{k}^{n}-C_{k-1}^{n}=\mathrm{E}^{n}\left[\left(N_{k}^{n}\right)^{2}-\left(N_{k-1}^{n}\right)^{2} \mid \mathcal{F}_{k-1}^{n}\right] \\
& =\mathrm{E}^{n}\left[\left(\Delta N_{k}^{n}\right)^{2} \mid \mathcal{F}_{k-1}^{n}\right]=\operatorname{var}^{n}\left[\Delta N_{k}^{n} \mid \mathcal{F}_{k-1}^{n}\right] .
\end{aligned}
$$

(Here and in the sequel, we use the notation $\Delta Y_{k}:=Y_{k}-Y_{k-1}$ for the increments of a discretetime process $Y$.) The first (and most laborious) step is thus to find the process $\Gamma^{n}$.

For ease of notation, in the subsequent computations we drop all sub- and superscripts $n$ and think of a fixed $n$. The only exceptions are in $\mu_{n}$ and $\sigma_{n}$, since we need to distinguish these from the constants $\mu$ and $\sigma$. We also omit all time arguments, since we first consider the case in which $a$ and $b$ do not depend on $t$.

To find a decomposition of $X$ like that in Proposition 1, an obvious first idea is to try the Doob decomposition $X=X_{0}+M+A$. Thus, we let

$$
\Delta A_{k}:=\mathrm{E}\left[\Delta X_{k} \mid \mathcal{F}_{k-1}\right]=\mu_{n}+\sigma_{n} \mathrm{E}\left[Z_{k} \mid \mathcal{F}_{k-1}\right]=\mu_{n}+\sigma_{n}\left(2 p\left(X_{k-1}, Z_{k-1}\right)-1\right),
$$

where we have used (1) and Condition 2. By using (6), (4), and (5), we obtain

$$
\Delta A_{k}=\mu \delta^{2}+\sigma b\left(X_{k-1}\right) \delta^{2}+\sigma_{n} Z_{k-1} a\left(X_{k-1}\right)+O\left(\delta^{2+\beta}\right) .
$$


To proceed with the computation of $\Delta A_{k}$, the simplest (but overly naive) way is to use a Taylor expansion of $a\left(X_{k-1}\right)$ around $X_{k-2}$, multiply the result by $\sigma_{n} Z_{k-1}$, and simplify. If we do this, on the right-hand side of (18) we obtain a term $a\left(X_{k-2}\right) \Delta A_{k-1}$, while $\Delta A_{k}$ remains on the left-hand side. Asymptotically, (18) thus produces an expression for $(1-a) \mathrm{d} A$ whereas we would like to have $\mathrm{d} A$ itself. Hence, it seems useful to divide by $1-a$ before doing more computations.

We have deliberately not given any details in the preceding reasoning, since its only purpose is to provide the motivation for our next step. The upshot is that we now Taylor expand the ratio $a\left(X_{k-1}\right) /\left(1-a\left(X_{k-1}\right)\right)$. Using (9), (7), (1), (4), and (5) yields

$$
\frac{a\left(X_{k-1}\right)}{1-a\left(X_{k-1}\right)}=\frac{a\left(X_{k-2}\right)}{1-a\left(X_{k-2}\right)}+\frac{a^{\prime}\left(X_{k-2}\right)}{\left(1-a\left(X_{k-2}\right)\right)^{2}} \Delta X_{k-1}+O\left(\delta^{1+\beta}\right)
$$

the error term comes from evaluating the derivative at $X_{k-2}$ instead of at a point between $X_{k-2}$ and $X_{k-1}$, and we have used the fact that $\left|\Delta X_{k-1}\right|=O(\delta)$. Now, by multiplying (19) by $\sigma_{n} Z_{k-1}=\Delta X_{k-1}-\mu_{n}$ and using (4), (5), and (1), we obtain

$$
\sigma_{n} Z_{k-1} \frac{a\left(X_{k-1}\right)}{1-a\left(X_{k-1}\right)}=\frac{a\left(X_{k-2}\right)}{1-a\left(X_{k-2}\right)}\left(\Delta X_{k-1}-\mu \delta^{2}\right)+\frac{a^{\prime}\left(X_{k-2}\right)}{\left(1-a\left(X_{k-2}\right)\right)^{2}} \sigma^{2} \delta^{2}+O\left(\delta^{2+\beta}\right) .
$$

Substituting this into (18) and using $\Delta X=\Delta M+\Delta A$ and the identity $a /(1-a)=$ $1 /(1-a)-1$ gives

$$
\begin{aligned}
\frac{\Delta X_{k}}{1-a\left(X_{k-1}\right)}= & \frac{\Delta M_{k}}{1-a\left(X_{k-1}\right)}+\frac{1}{1-a\left(X_{k-2}\right)} \Delta X_{k-1}-\Delta X_{k-1} \\
& +\frac{\mu+\sigma b\left(X_{k-1}\right)}{1-a\left(X_{k-1}\right)} \delta^{2}+\mu \delta^{2}\left(1-\frac{1}{1-a\left(X_{k-2}\right)}\right)+\frac{a^{\prime}\left(X_{k-2}\right)}{\left(1-a\left(X_{k-2}\right)\right)^{2}} \sigma^{2} \delta^{2} \\
& +O\left(\delta^{2+\beta}\right) .
\end{aligned}
$$

Lemma 1. Define the martingale $N$ by

$$
N_{m}:=\sum_{k=1}^{m} \frac{\Delta M_{k}}{1-a\left(X_{k-1}\right)}, \quad m=0,1, \ldots, n,
$$

and the process $\Gamma$ by $\Gamma:=X-X_{0}-N$. Then we have

$$
\Gamma_{m}=\sum_{k=1}^{m}\left(\mu+\frac{\sigma b\left(X_{k-1}\right)}{1-a\left(X_{k-1}\right)}+\frac{\sigma^{2} a^{\prime}\left(X_{k-1}\right)}{\left(1-a\left(X_{k-1}\right)\right)^{2}}\right) \delta^{2}+O\left(\delta^{\beta}\right), \quad m=0,1, \ldots, n .
$$

Proof. We start from (20), which we rewrite as

$$
\begin{aligned}
\Delta X_{k-1}= & \frac{\Delta M_{k}}{1-a\left(X_{k-1}\right)}+\left(\mu+\frac{\sigma b\left(X_{k-1}\right)}{1-a\left(X_{k-1}\right)}+\frac{\sigma^{2} a^{\prime}\left(X_{k-1}\right)}{\left(1-a\left(X_{k-1}\right)\right)^{2}}\right) \delta^{2} \\
& +\left(\frac{\Delta X_{k-1}}{1-a\left(X_{k-2}\right)}-\frac{\Delta X_{k}}{1-a\left(X_{k-1}\right)}\right)+\mu \delta^{2}\left(\frac{1}{1-a\left(X_{k-1}\right)}-\frac{1}{1-a\left(X_{k-2}\right)}\right) \\
& +\sigma^{2} \delta^{2}\left(\frac{a^{\prime}\left(X_{k-2}\right)}{\left(1-a\left(X_{k-2}\right)\right)^{2}}-\frac{a^{\prime}\left(X_{k-1}\right)}{\left(1-a\left(X_{k-1}\right)\right)^{2}}\right)+O\left(\delta^{2+\beta}\right) .
\end{aligned}
$$


Owing to (7) and the fact that

$$
\left|\Delta X_{k}\right|=O(\delta),
$$

the errors we make by summing over $k$ from 1 or 2 to $m$ or $m+1$ are $O(\delta)$. The third, fourth, and fifth terms in (23) all yield telescoping series whose sums are $O(\delta)$ due to (7) and (8). Finally, since we sum at most $n$ terms and $\delta=\delta_{n}=1 / \sqrt{n}$, the sum of the terms comprising $O\left(\delta^{2+\beta}\right)$ is $O\left(\delta^{\beta}\right)$. Hence, the assertion follows.

Lemma 2. Define the martingale $N$ by (21) and the increasing process $C$ by

$$
C_{m}:=\sum_{k=1}^{m} \frac{\operatorname{var}\left[\Delta M_{k} \mid \mathcal{F}_{k-1}\right]}{\left(1-a\left(X_{k-1}\right)\right)^{2}}, \quad m=0,1, \ldots, n .
$$

Then $N^{2}-C$ is a martingale and

$$
C_{m}=\sum_{k=1}^{m} \sigma^{2} \frac{1+a\left(X_{k-1}\right)}{1-a\left(X_{k-1}\right)} \delta^{2}+O\left(\delta^{\beta}\right), \quad m=0,1, \ldots, n .
$$

Proof. Since $N$ is a martingale, it is clear from (21) that $N^{2}-C$, with $C$ from (24), is also a martingale. To prove (25), it is enough to show that

$$
\operatorname{var}\left[\Delta M_{k} \mid \mathcal{F}_{k-1}\right]=\operatorname{var}\left[\Delta X_{k} \mid \mathscr{F}_{k-1}\right]=\left(1-a^{2}\left(X_{k-1}\right)\right) \sigma^{2} \delta^{2}+O\left(\delta^{2+\beta}\right),
$$

and, if we note that (1), Condition 2, and (6) yield

$$
\begin{aligned}
\operatorname{var}\left[\Delta X_{k} \mid \mathcal{F}_{k-1}\right] & =\sigma_{n}^{2} \operatorname{var}\left[Z_{k} \mid \mathcal{F}_{k-1}\right] \\
& =4 \sigma_{n}^{2} p\left(X_{k-1}, Z_{k-1}\right)\left(1-p\left(X_{k-1}, Z_{k-1}\right)\right) \\
& =\sigma_{n}^{2}\left(1-Z_{k-1}^{2} a^{2}\left(X_{k-1}\right)+O(\delta)\right),
\end{aligned}
$$

we see that (26) follows from (4).

Having found the processes $N, \Gamma$, and $C$, we now want to verify that they satisfy the assumptions of Proposition 1. We thus reinstate the indices $n$ and define the functions

$$
\gamma(x):=\mu+\frac{\sigma b(x)}{1-a(x)}+\frac{\sigma^{2} a^{\prime}(x)}{(1-a(x))^{2}}, \quad c(x):=\sigma^{2} \frac{1+a(x)}{1-a(x)} .
$$

These appear in $L$ and as coefficients of $\delta_{n}^{2}$ in (22) and (25), and are bounded due to (7) and (8). The conditions (13)-(15) are easy to check. In fact, (1), (4), and (5) give

$$
\sup _{0 \leq t \leq 1}\left|X_{t}^{(n)}-X_{t-}^{(n)}\right|=\max _{k=1, \ldots, n}\left|\Delta X_{k}^{n}\right|=O\left(\delta_{n}\right),
$$

where, recall, $X^{(n)}$ is the piecewise-constant interpolation of $X^{n}$. In the same way, we obtain

$$
\sup _{0 \leq t \leq 1}\left|\Gamma_{t}^{(n)}-\Gamma_{t-}^{(n)}\right|+\sup _{0 \leq t \leq 1}\left|C_{t}^{(n)}-C_{t-}^{(n)}\right|=O\left(\delta_{n}^{\beta}\right),
$$

since $\gamma$ and $c$ are bounded. 
Lemma 3. For $\Gamma^{(n)}, C^{(n)}$, and $\gamma$ and c defined by (22), (25), and, respectively, (27), conditions (16) and (17) are satisfied.

Proof. Since the argument is the same in both cases, we prove only (16). For $t \in\left[t_{m}^{n}, t_{m+1}^{n}\right)$, using both the fact that $X^{(n)}$ and $\Gamma^{(n)}$ are piecewise constant and Lemma 1 yields

$$
\begin{aligned}
\left|\Gamma_{t}^{(n)}-\int_{0}^{t} \gamma\left(X_{s}^{(n)}\right) \mathrm{d} s\right| & =\left|\Gamma_{m}^{n}-\sum_{k=1}^{m} \int_{t_{k-1}^{n}}^{t_{k}^{n}} \gamma\left(X_{s}^{(n)}\right) \mathrm{d} s-\int_{t_{m}^{n}}^{t} \gamma\left(X_{s}^{(n)}\right) \mathrm{d} s\right| \\
& \leq O\left(\delta_{n}^{\beta}\right)+\sum_{k=1}^{m}\left|\gamma\left(X_{k-1}^{n}\right) \delta_{n}^{2}-\int_{t_{k-1}^{n}}^{t_{k}^{n}} \gamma\left(X_{k-1}^{n}\right) \mathrm{d} s\right|+\|\gamma\|_{\infty} \delta_{n}^{2} \\
& =O\left(\delta_{n}^{\beta}\right),
\end{aligned}
$$

and, since this is uniform in $t,(16)$ follows.

Lemmas 1, 2, and 3 imply that, in the setting of Theorem 1, all the assumptions of Proposition 1 are satisfied. Thus, $\left(X^{(n)}\right)_{n \in \mathbb{N}}$ converges in distribution to the solution to the martingale problem for $(G, v)$, which, in view of (27), is the same as the solution to (12). This completes the proof of Theorem 1 for the case in which $a$ and $b$ do not depend on $t$.

Before we start on the time-dependent case, we introduce some further notation.

Definition 1. Consider functions $f:[0,1] \times \mathbb{R} \rightarrow \mathbb{R},(t, x) \mapsto f(t, x)$. For $\beta, \varepsilon \in(0,1)$, $H^{\beta / 2, \varepsilon}$ is the space of all such $f$ that are continuous and bounded; $\operatorname{Hölder}\left(\frac{1}{2} \beta\right)$-continuous in $t$, uniformly in $x$; and Hölder $(\varepsilon)$-continuous in $x$, uniformly in $t . H^{(1+\beta) / 2,1+\varepsilon}$ is the space of all $f$ that are continuous and bounded; $\operatorname{Hölder}\left(\frac{1}{2}(1+\beta)\right)$-continuous in $t$, uniformly in $x$; and differentiable in $x$ such that $f^{\prime}(t, x)=(\partial / \partial x) f(t, x)$ is bounded; Hölder $\left(\frac{1}{2} \beta\right)$-continuous in $t$, uniformly in $x$; and $\operatorname{Hölder}(\varepsilon)$-continuous in $x$, uniformly in $t . H^{\beta / 2,1+\varepsilon}$ is the space of all $f$ that are continuous and bounded; $\operatorname{Hölder}\left(\frac{1}{2} \beta\right)$-continuous in $t$, uniformly in $x$; and differentiable in $x$ such that $f^{\prime}(t, x)=(\partial / \partial x) f(t, x)$ is bounded; Hölder $\left(\frac{1}{2} \beta\right)$-continuous in $t$, uniformly in $x$; and Hölder $(\varepsilon)$-continuous in $x$, uniformly in $t$.

Suppose now that $a$ and $b$ are allowed to depend on $t$. We can then prove Theorem 1 using almost the same arguments as above if we additionally assume that

$$
a \in H^{(1+\beta) / 2,1+\beta} \quad \text { and } \quad b \in H^{\beta / 2, \beta} \text {. }
$$

(See below for comments on how this is related to the original assumptions of Theorem 1.) In fact, (29) guarantees that we can again do a Taylor expansion (now in both $t$ and $x$ ) for $f(t, x):=$ $a(t, x) /(1-a(t, x))$ and obtain (19) with all arguments $X_{k-j}$ replaced by $\left(t_{k-j+1}^{n}, X_{k-j}^{n}\right)$. The crucial point here is that, under (29), $f(t, x)$ is also Hölder $\left(\frac{1}{2}(1+\beta)\right)$-continuous in $t$. Once we have (19) in this time-dependent form, the same argument using telescoping series still yields Lemma 1. Lemma 2 and (13)-(15) are proved as before, and the only other change occurs in the proof of Lemma 3. First, note that, due to (29), both $c$ and $\gamma$ are $\operatorname{Hölder}\left(\frac{1}{2} \beta\right)$-continuous in $t$, uniformly in $x$. Rewriting (28) in time-dependent form gives

$$
\begin{aligned}
\left|\Gamma_{t}^{(n)}-\int_{0}^{t} \gamma\left(s, X_{s}^{(n)}\right) \mathrm{d} s\right| & \leq O\left(\delta_{n}^{\beta}\right)+\sum_{k=1}^{m}\left|\gamma\left(t_{k}^{n}, X_{k-1}^{n}\right) \delta_{n}^{2}-\int_{t_{k-1}^{n}}^{t_{k}^{n}} \gamma\left(s, X_{k-1}^{n}\right) \mathrm{d} s\right|+\|\gamma\|_{\infty} \delta_{n}^{2} \\
& \leq O\left(\delta_{n}^{\beta}\right)+\sum_{k=1}^{m} \int_{t_{k-1}^{n}}^{t_{k}^{n}}\left|\gamma\left(t_{k}^{n}, X_{k-1}^{n}\right)-\gamma\left(s, X_{k-1}^{n}\right)\right| \mathrm{d} s
\end{aligned}
$$


and each integrand is $O\left(\left(\delta_{n}^{2}\right)^{\beta / 2}\right)=O\left(\delta_{n}^{\beta}\right)$. Thus, all of the at most $n$ summands are $O\left(\delta_{n}^{2+\beta}\right)$ because $t_{k}^{n}-t_{k-1}^{n}=(1 / n)=\delta_{n}^{2}$. This holds uniformly in $t$; thus, we obtain

$$
\sup _{0 \leq t \leq 1}\left|\Gamma_{t}^{(n)}-\int_{0}^{t} \gamma\left(s, X_{s}^{(n)}\right) \mathrm{d} s\right| \leq O\left(\delta_{n}^{\beta}\right),
$$

which clearly implies (16). The argument for (17) is completely analogous. Finally, we note that Proposition 1 can easily be extended to the time-dependent form we need here. This shows how to prove Theorem 1 under the additional assumption (29).

Remark 2. Comparing Condition 3 with (29) reveals that the latter is only a quantitative strengthening of (11); we replace mere continuity of $a^{\prime}(t, x)$ and $b(t, x)$ in $t$ by Hölder $\left(\frac{1}{2} \beta\right)$ continuity in $t$, uniformly in $x$. The above sketch of the proof also shows how this is exploited.

If $a$ and $b$ satisfy the assumptions of Condition 3 but not (29), the preceding proof no longer seems to work. The reason is that the Taylor expansion in (19) can then only be made in $x$ alone. Hence, it involves arguments $\left(t_{k}^{n}, X_{k-1}^{n}\right)$ on the left-hand side and $\left(t_{k}^{n}, X_{k-2}^{n}\right)$ (instead of $\left.\left(t_{k-1}^{n}, X_{k-2}^{n}\right)\right)$ on the right-hand side, and the telescoping series argument thus no longer works. So far, we have not been able to overcome the resulting complications in (20).

Nevertheless, Theorem 1 is true in the generality given here. An alternative proof can be found in Gruber (2004), but it is rather long and technically involved. We have therefore decided to prove Theorem 1 here only under slightly less general assumptions. Very briefly, the proof in Gruber (2004) can be summarized as follows. In a first step, assuming that (4), (5), (7), and (8) hold, the sequence $\left(X^{(n)}\right)_{n \in \mathbb{N}}$ is shown to be tight in $D[0,1]$ by deriving precise bounds on product moments of the increments of $X^{n}$ and then employing techniques from Billingsley (1968, pp. 87-95) for controlling the fluctuations of partial sums of not necessarily independent nor identically distributed random variables. The second step then shows that the weak limit of any convergent subsequence of $\left(X^{(n)}\right)$ solves the martingale problem for $(L, v)$. Since the correlation between two successive increments of $X^{(n)}$ does not vanish as $n \rightarrow \infty$, this requires a careful consideration of conditional moments on a time scale of $O\left(\delta_{n}\right)$. By further refining the arguments of Gruber (2004), we can probably even abandon the Hölder continuity assumptions in the $x$-variable as well.

Remark 3. If we want to allow $a$ and $b$ in (6) to depend on $n$, we can do so as follows. For $f \in\{a, b\}$, replace $f(t, x)$ by

$$
f_{n}(t, x)=f(t, x)+\xi_{n}^{f}
$$

where the constants $\left(\xi_{n}^{f}\right)_{n \in \mathbb{N}}$ satisfy

$$
\xi_{n}^{f}=O\left(\delta_{n}^{\beta}\right) \quad \text { for some } \beta \in(0,1) .
$$

All other conditions are unchanged. If we have (29), we can still prove Theorem 1 almost as above by replacing $f$ with $f_{n}$; the only change is that the integrand in (30) becomes

$$
\left|\gamma_{n}\left(t_{k}^{n}, X_{k-1}^{n}\right)-\gamma\left(s, X_{k-1}^{n}\right)\right| \leq\left|\left(\gamma_{n}-\gamma\right)\left(t_{k}^{n}, X_{k-1}^{n}\right)\right|+\left|\gamma\left(t_{k}^{n}, X_{k-1}^{n}\right)-\gamma\left(s, X_{k-1}^{n}\right)\right| .
$$

However, combining (31) with Condition 2 easily yields $\left\|\gamma_{n}-\gamma\right\|_{\infty} \leq O\left(\delta_{n}^{\beta}\right)$ as well as $\left\|c_{n}-c\right\|_{\infty} \leq O\left(\delta_{n}^{\beta}\right)$; this allows us to finish the proof as before. 


\section{Examples and applications}

In this section, we present three situations in which Theorem 1 can be useful.

\subsection{Option pricing with a large investor}

Our first example actually provided the motivation and set-up for Theorem 1 . However, its details are too involved for a full presentation here. Hence, we only sketch the main ideas, and refer the reader to Gruber (2004) and forthcoming work for more information.

The basic question is easy to explain. A large investor in a financial market wants to price an option, and 'large' means that the hedging strategy he plans to construct for replicating the option has an impact on the price process of the underlying asset (stock, say). What, then, are the value of the option and the resulting stock price evolution?

To formalize this situation, we start with a discrete-time model in which uncertainty is generated by a binary random walk $X^{n}$; this describes some uncertain fundamental quantities in the financial market. We construct a mechanism for the price formation of the stock and, thus, obtain its price process for any exogenously given strategy of the large investor. However, the hedging strategy we want must be determined endogenously, since it must replicate a pay-off on the given stock. It will thus be given via a fixed-point argument, and the resulting transition probabilities for the evolution of uncertainty will be obtainable only from an implicit equation.

It turns out that, under smoothness conditions on the pay-off, the hedging function for the option can be recursively described backwards in time. This yields a difference equation whose continuous-time limit is a nonlinear partial differential equation. Under suitable assumptions, we can show that this partial differential equation has a unique solution $\varphi$, and that the transition probabilities in discrete time can be described as in (6), in terms of $\varphi$. More precisely, this is true for the transition probabilities of $X^{n}$ under a measure that turns both the stock price process resulting for the large investor and his valuation process for the option into martingales. Using Theorem 1 then produces continuous-time models for option valuation with a large investor. In particular, this provides new insights into the precise impact of the model chosen for the market mechanism.

In a little more detail, the price formation mechanism is described by two ingredients: a reaction function $\psi(t, x, \vartheta)$ of time, $t$, the current fundamental value, $x$, and the stock holding, $\vartheta$, of the large investor, and a measure $\varrho$ that models the timing in forming the price at which the large investor can trade. The diffusion coefficient of the limit process $X$ is then given by

$$
\sigma_{\varphi}^{2}(t, x)=\frac{\psi_{x}(t, x, \varphi(t, x))+2 d(\varrho) \psi_{\vartheta}(t, x, \varphi(t, x)) \varphi_{x}(t, x)}{\psi_{x}(t, x, \varphi(t, x))+\psi_{\vartheta}(t, x, \varphi(t, x)) \varphi_{x}(t, x)}
$$

with $d(\varrho)=\int_{\mathbb{R}} z \varrho(\mathrm{d} z)-\frac{1}{2}$; see Theorem 4.4 and Equation (4.2.22) of Gruber (2004). Here a subscript denotes partial differentiation with respect to the relevant argument.

Even without going into any further detail, we can explain why generalized correlated random walks come up in this context. It is well known that the value of an option whose pay-off at time 1 is of the form $h\left(X_{1}\right)$ can usually be obtained as a function $v\left(t, X_{t}\right)$, and that the corresponding hedging strategy is given in terms of the derivative $(\partial / \partial x) v\left(t, X_{t}\right)$. If we now consider a large investor in discrete time, the price formation mechanism involves not only the current price $X_{k}^{n}$, but, via the strategy's impact, also the increment $\Delta X_{k}^{n}$. Hence, the transition probabilities of $X^{n}$ take the form imposed by Condition 2 and we no longer have a simple Markovian structure for $X^{n}$ or $Z^{n}$ alone. 


\subsection{Option pricing under transaction costs}

Consider a financial market with a bank account $B$ and a stock $S$ traded with transaction costs as follows. If the (nominal) stock price at time $t$ is $S_{t}$, a share can be bought for $\left(1+\kappa^{\mathrm{b}}\right) S_{t}$ but sold only for $\left(1-\kappa^{\mathrm{s}}\right) S_{t}$, where $\kappa^{\mathrm{b}}, \kappa^{\mathrm{s}} \in[0,1)$. What is then a reasonable price for a European call option on $S$ ?

This question has been studied and answered in the well-known Cox-Ross-Rubinstein model, in which the stock price process is given by a geometric binary random walk. More precisely, suppose that $S^{n}=\left(S_{k}^{n}\right)_{k=0,1, \ldots, n}$ is given by

$$
S_{k}^{n}=S_{0}^{n} \exp \left(X_{k}^{n}\right)=S_{0}^{n} \exp \left(\sum_{j=1}^{k} \Delta X_{j}^{n}\right),
$$

where, for each $n$, the $\log$-returns $\Delta X_{j}^{n}=\log \left(S_{j}^{n} / S_{j-1}^{n}\right)$ take the value $u_{n}$ or $d_{n}$ while the bank account evolves according to $B_{k}^{n}=\exp \left(k r_{n}\right)$ for $k=0,1, \ldots, n$, with $u_{n}>r_{n}>d_{n}$. Boyle and Vorst (1992) showed that there exists a unique strategy that is self-financing, inclusive of transaction costs, and has a final wealth, $\left(S_{n}^{n}-K\right)^{+}=h\left(S_{n}^{n}\right)$, equal to the pay-off of the call. The option's value is thus the initial cost for this strategy, and it turns out that it can be computed as the expectation of the discounted pay-off $h\left(S_{n}^{n}\right) / B_{n}^{n}$ under a probability measure $\mathrm{P}_{n}^{*}$ under which the $\Delta X_{k}^{n}$ form a Markov chain. By using the central limit theorem and an appropriate scaling of parameters, Boyle and Vorst (1992) also showed that this option pricing formula converges to the Black-Scholes formula, but with a higher variance than in the case of no transaction costs. For a general pay-off function $h$, Kusuoka (1995) gave an expression for the limit of the superreplication price for $h\left(S_{n}^{n}\right)$; his results also showed that the superreplication price coincides with the above replication price if $h$ is convex and monotonic.

As in Section 2, $S^{(n)}$ denotes the piecewise-constant interpolation of $S^{n}$ on [0,1]. While convergence of option prices is a result on the one-dimensional marginal distributions of $S^{(n)}$ at time 1 , we are here interested in a weak convergence result for the whole distribution of the process $S^{(n)}$. In the case without transaction costs, the $\Delta X_{k}^{n}$ are independent under $\mathrm{P}_{n}^{*}$ and it follows from Donsker's theorem that the Cox-Ross-Rubinstein models converge weakly to the Black-Scholes model of geometric Brownian motion; see Chapter 22 of Duffie (1988) for a detailed account. The case with transaction costs has been treated in the (unpublished, German) diploma thesis of Opitz (1999); we now show how to deduce the results from Theorem 1.

Let us first specify the parameters. As in Section 2, we work on $[0,1]$ and write $\delta_{n}=1 / \sqrt{n}$. For each $n$, the random variables $\Delta X_{k}^{n}=\mu_{n}+\sigma_{n} Z_{k}^{n}$ take either of the two values

$$
u_{n}=\mu \delta_{n}^{2}+\sigma \delta_{n} \quad \text { and } \quad d_{n}=\mu \delta_{n}^{2}-\sigma \delta_{n}, \quad \text { with } \mu \in \mathbb{R} \text { and } \sigma>0 ;
$$

thus, we have

$$
\sigma_{n}=\sigma \delta_{n}, \quad \mu_{n}=\mu \delta_{n}^{2} .
$$

The bank account is determined by

$$
r_{n}=\varrho \delta_{n}^{2}, \quad \text { with } \varrho \in \mathbb{R},
$$

and transaction costs are specified by

$$
\kappa_{n}^{\mathrm{x}}=\kappa^{\mathrm{x}} \delta_{n}, \quad \text { with } 0 \leq \kappa^{\mathrm{x}}<1 \text { for } \mathrm{x} \in\{\mathrm{b}, \mathrm{s}\} .
$$


The probability measure $\mathrm{P}_{n}^{*}$ is defined by its transition probabilities

$$
\mathrm{P}_{n}^{*}\left[Z_{k}^{n}=1 \mid \mathcal{F}_{k-1}^{n}\right]=\mathrm{P}_{n}^{*}\left[Z_{k}^{n}=1 \mid Z_{k-1}^{n}\right],
$$

with

$$
\begin{gathered}
\mathrm{P}_{n}^{*}\left[Z_{k}^{n}=1 \mid Z_{k-1}^{n}=1\right]=p_{n, U}^{*}:=\frac{\mathrm{e}^{r_{n}}\left(1+\kappa_{n}^{\mathrm{b}}\right)-\mathrm{e}^{d_{n}}\left(1-\kappa_{n}^{\mathrm{s}}\right)}{\mathrm{e}^{u_{n}}\left(1+\kappa_{n}^{\mathrm{b}}\right)-\mathrm{e}^{d_{n}}\left(1-\kappa_{n}^{\mathrm{s}}\right)}, \\
\mathrm{P}_{n}^{*}\left[Z_{k}^{n}=1 \mid Z_{k-1}^{n}=-1\right]=p_{n, D}^{*}:=\frac{\mathrm{e}^{r_{n}}\left(1-\kappa_{n}^{\mathrm{s}}\right)-\mathrm{e}^{d_{n}}\left(1-\kappa_{n}^{\mathrm{s}}\right)}{\mathrm{e}^{u_{n}}\left(1+\kappa_{n}^{\mathrm{b}}\right)-\mathrm{e}^{d_{n}}\left(1-\kappa_{n}^{\mathrm{s}}\right)} ;
\end{gathered}
$$

asymptotically, the initial distribution of $Z_{0}^{n}$ plays no role. By writing (37) as

$$
\mathrm{P}_{n}^{*}\left[Z_{k}^{n}=1 \mid Z_{k-1}^{n}\right]=\frac{1}{2}\left(p_{n, U}^{*}+p_{n, D}^{*}+Z_{k-1}^{n}\left(p_{n, U}^{*}-p_{n, D}^{*}\right)\right),
$$

we obtain the following lemma.

Lemma 4. In the above setting, we have

$$
\mathrm{P}_{n}^{*}\left[Z_{k}^{n}=1 \mid \mathcal{F}_{k-1}^{n}\right]=p_{n}^{*}\left(k, Z_{k-1}^{n}\right),
$$

with

$$
p_{n}^{*}(k, z)=\frac{1}{2}\left(1+z \lambda_{n}+\delta_{n} \varphi_{n}\right)+O\left(\delta_{n}^{2}\right)
$$

where

$$
\begin{array}{ll}
\lambda_{n}:=\lambda+O\left(\delta_{n}\right):=\frac{\kappa^{\mathrm{b}}+\kappa^{\mathrm{s}}}{2 \sigma+\kappa^{\mathrm{b}}+\kappa^{\mathrm{s}}}+O\left(\delta_{n}\right) & \text { as } n \rightarrow \infty, \\
\varphi_{n}:=\varphi+O\left(\delta_{n}\right):=\frac{2(\varrho-\mu)-\left(\kappa^{\mathrm{b}}+\kappa^{\mathrm{s}}+\sigma\right) \sigma}{2 \sigma+\kappa^{\mathrm{b}}+\kappa^{\mathrm{s}}}+O\left(\delta_{n}\right) & \text { as } n \rightarrow \infty .
\end{array}
$$

Proof. We omit the proof, since it consists of straightforward computations.

If we compare (38) with (6), we see that the present situation does not exactly fit into the setting of Theorem 1. However, the extension explained in Remark 3 allows us to derive the following result, first obtained by Opitz (1999).

Theorem 2. Suppose that $S^{(n)}$ is the piecewise-constant interpolation of $S^{n}$ defined by (32) with the parameters specified by (33)-(36). Let $\mathrm{P}_{n}^{*}$ be given by (37) and suppose that $S_{0}^{n} \rightarrow S_{0}$ as $n \rightarrow \infty$. Then $\left(S^{(n)}\right)_{n \in \mathbb{N}}$ converges in distribution under $\mathrm{P}_{n}^{*}$ to

$$
S_{t}=S_{0} \exp \left(\bar{\sigma} W_{t}+\left(\varrho-\frac{1}{2} \bar{\sigma}^{2}\right) t\right), \quad 0 \leq t \leq 1,
$$

for a Brownian motion $W$, where

$$
\bar{\sigma}^{2}:=\sigma\left(\sigma+\kappa^{\mathrm{b}}+\kappa^{\mathrm{s}}\right)=\sigma^{2}\left(1+\frac{\kappa^{\mathrm{b}}+\kappa^{\mathrm{s}}}{\sigma}\right) .
$$

Proof. By letting $a_{n}(t, x):=\lambda_{n}$ and $b_{n}(t, x):=\varphi_{n}$, from (27), (39), and (40) we find that

$$
\gamma(t, x)=\mu+\frac{\sigma \varphi}{1-\lambda}=\rho-\frac{1}{2} \bar{\sigma}^{2}, \quad c(t, x)=\sigma^{2} \frac{1+\lambda}{1-\lambda}=\bar{\sigma}^{2} .
$$

Hence, the result follows by applying the $n$-dependent extension of Theorem 1 to $X^{(n)}=$ $\log \left(S^{(n)} / S_{0}^{(n)}\right)$, under $\mathrm{P}_{n}^{*}$, and using the continuous mapping theorem.

Remark 4. Of course, Theorem 2 implies the convergence result of Boyle and Vorst (1992). 


\subsection{Approximating diffusions by regular recombining binomial trees}

In many situations it is necessary to approximate a diffusion process $X$ given by a stochastic differential equation by a discrete-time process $X^{n}$, in order to compute approximations for some functionals of $X$. A typical example occurs in mathematical finance when it is desired to compute values and hedging strategies for options written on $X$. The value function $v$ is usually given by some expectation, and the hedging strategy involves the derivative of $v$. To approximate this efficiently by discrete differences, we would like to have some control over the values taken by $X^{n}$. In particular, computations are often more efficient if these values lie on a regular grid. Our next application of Theorem 1 shows how this can be achieved. For those readers who skipped the proof of Theorem 1, we remark that the Hölder spaces $H^{{ }^{\circ},}$ are defined in Definition 1.

Theorem 3. Suppose that $\hat{\sigma} \in H^{\beta / 2,1+\beta}$ and $\hat{\mu} \in H^{\beta / 2, \beta}$, with $\beta \in(0,1)$. If $\hat{\sigma}:[0,1] \times$ $\mathbb{R} \rightarrow \mathbb{R}$ is bounded away from 0 , uniformly in $t$ and $x$, then there exists a sequence of generalized correlated random walks $X^{n}$, as in Section 2, whose corresponding piecewiseconstant interpolations $X^{(n)}$ converge in distribution to the process $X$ given by the stochastic differential equation

$$
\mathrm{d} X_{t}=\hat{\mu}\left(t, X_{t}\right) \mathrm{d} t+\hat{\sigma}\left(t, X_{t}\right) \mathrm{d} W_{t} .
$$

Proof. If we define the functions $a$ and $b$ by

$$
a(t, x):=\frac{\hat{\sigma}^{2}(t, x)-1}{\hat{\sigma}^{2}(t, x)+1} \quad \text { and } \quad b(t, x):=2 \frac{\hat{\mu}(t, x)-\hat{\sigma}(t, x) \hat{\sigma}^{\prime}(t, x)}{\hat{\sigma}^{2}(t, x)+1},
$$

then a straightforward calculation yields

$$
\frac{1+a(t, x)}{1-a(t, x)}=\hat{\sigma}^{2}(t, x) \quad \text { and } \quad \frac{b(t, x)}{1-a(t, x)}+\frac{a^{\prime}(t, x)}{(1-a(t, x))^{2}}=\hat{\mu}(t, x) .
$$

We now define $X^{n}$ by (2) with $X_{0}^{n}=X_{0}, Z_{0}^{n}=1$, and the parameters $\mu_{n} \equiv 0$ and $\sigma_{n}=\delta_{n}=$ $1 / \sqrt{n}$, and the transition function $p_{n}$ as in (6) with $a$ and $b$ as above. The assertion then follows directly from Theorem 1 with $\mu=0$ and $\sigma=1$.

To see why Theorem 3 is useful, assume that $\hat{\mu}(t, x)$ or $\hat{\sigma}(t, x)$ is not constant in the space variable $x$. The most straightforward way of approximating $X$ by a binomial process $\tilde{X}^{n}$ is to set

$$
\mathrm{P}^{n}\left[\tilde{X}_{k}^{n}=\tilde{X}_{k-1}^{n}+\hat{\mu}\left(t_{k}^{n}, \tilde{X}_{k-1}^{n}\right) \delta_{n}^{2} \pm \hat{\sigma}\left(t_{k}^{n}, \tilde{X}_{k-1}^{n}\right) \delta_{n} \mid \mathcal{F}_{k-1}^{n}\right]=\frac{1}{2} .
$$

However, this leads to a nonrecombining tree for the paths of $\tilde{X}^{n}$, since the value after $\tilde{X}^{n}$ makes an upward move followed by a downward move need not coincide with the value attained if the steps are made in reverse order. A common method of constructing a recombining binomial approximation of the diffusion (41) is due to Nelson and Ramaswamy (1990). They first constructed a suitable transformation, $g(X)$, of $X$ with constant volatility, developed an approximation for $g(X)$ using a simple binomial process on a recombining tree, and then applied the inverse of $g$ to obtain a binomial approximation, $\bar{X}^{n}$, of $X$ itself. The paths of $\bar{X}^{n}$ are then still recombining, but the corresponding tree is compressed and stretched in space non-uniformly.

In the proof of Theorem 3, a generalized correlated random walk $X^{n}$ that satisfies $\delta_{n}=$ $\left|X_{k}^{n}-X_{k-1}^{n}\right|$ for all $k$ is now constructed. This means that the corresponding binomial tree is both recombining and homogeneous in space. The striking simplicity of this structure is a 
consequence of allowing the transition probabilities of $X^{n}$ to depend not only on $t_{k}^{n}$ and $X_{k}^{n}$, but also on the increment $\Delta X_{k}^{n}=Z_{k}^{n}$. It will be interesting to see if this can be used to improve calculation efficiency in financial models.

\section{Acknowledgements}

Financial support by the Deutsche Forschungsgemeinschaft through the Graduiertenkolleg 'Stochastische Prozesse und probabilistische Analysis' at the Technical University of Berlin is gratefully acknowledged. Part of this research was carried out within the 'Mathematical Methods in Financial Risk Management' project of the National Centre of Competence in Research 'Financial Valuation and Risk Management' research program (NCCR FINRISK), which is supported by the Swiss National Science Foundation. Parts of this paper were written in Cambridge, at the Isaac Newton Institute for Mathematical Sciences, and MS is grateful for financial support received there in connection with the programme 'Developments in Quantitative Finance'.

\section{References}

Billingsley, P. (1968). Convergence of Probability Measures. John Wiley, New York.

Boyle, P. P. And Vorst, T. (1992). Option replication in discrete time with transaction costs. J. Finance 47, $271-293$.

Chen, A. And Renshaw, E. (1994). The general correlated random walk. J. Appl. Prob. 31, 869-884.

Duffie, D. (1988). Security Markets. Stochastic Models. Academic Press, Boston, MA.

Ethier, S. ANd Kurtz, T. (1986). Markov Processes. Characterization and Convergence. John Wiley, New York.

Gruber, U. (2004). Convergence of binomial large investor models and general correlated random walks. Doctoral Thesis, Technical University of Berlin. Available at http://edocs.tu-berlin.de/diss/2004/gruber_urs.htm.

Horváth, L. AND SHAO, Q.-M. (1998). Limit distributions of directionally reinforced random walks. Adv. Math. 134, 367-383.

Kusuoka, S. (1995). Limit theorem on option replication cost with transaction costs. Ann. Appl. Prob. 5, $198-221$.

Mauldin, R. D., Monticino, M. And von Weizsäcker, H. (1996). Directionally reinforced random walks. Adv. Math. 117, 239-252.

Nelson, D. B. (1990). ARCH models as diffusion approximations. J. Econometrics 45, 7-38.

Nelson, D. B. and Ramaswamy, K. (1990). Simple binomial processes as diffusion approximations in financial models. Rev. Financial Studies 3, 393-430.

OpITZ, A. (1999). Zur Asymptotik der Bewertung von Optionen unter Transaktionskosten im Binomialmodell. Diploma Thesis, Technical University of Berlin.

Renshaw, E. and Henderson, R. (1981). The correlated random walk. J. Appl. Prob. 18, 403-414.

Stroock, D. W. and Varadhan, S. R. S. (1979). Multidimensional Diffusion Processes. Springer, New York.

Szász, D. AND Tóth, B. (1984). Persistent random walks in a one-dimensional random environment. J. Statist. Phys. 37, 27-38. 